\title{
Conformational and vibrational study of platinum(II) anticancer drugs: cis-diamminedichloroplatinum (II) as a case study
}

\author{
Ana M. Amado, ${ }^{\text {a) }}$ Sónia M. Fiuza, Maria P. M. Marques, and Luis A. E. Batista de Carvalho \\ Química-Física Molecular, Departamento de Química, FCTUC, Universidade de Coimbra, P-3004-535 \\ Coimbra, Portugal
}

(Received 6 July 2007; accepted 29 August 2007; published online 12 November 2007)

\begin{abstract}
A conformational and vibrational analysis of cisplatin [cis-diamminedichloro-platinum(II)] (cDDP) is reported. Several theory methods (from Hartree-Fock to Møller-Plesset and density functional theory) combined with different all-electron basis sets are evaluated, in view of determining the best suited strategy for accurately representing this molecule. This choice is based on the best compromise between accuracy and computational requirements. Different scaling models of the cDDP vibrational modes were tested for obtaining the best scaling factors to be used in this type of inorganic systems. The structural parameters and vibrational results predicted by the calculations are compared with the corresponding experimental data, namely, x-ray structure, and Raman and inelastic neutron scattering spectra. Finally, a complete assignment of the cDDP vibrational spectra is presented. (C) 2007 American Institute of Physics. [DOI: 10.1063/1.2787528]
\end{abstract}

\section{INTRODUCTION}

Cisplatin [cis-diamminedichloroplatinum(II), cDDP] is still among the most widely used drugs in cancer chemotherapy. It displays significant activity against several types of neoplastic disorders, with special success against testicular and ovarian cancers. Despite this remarkable cytotoxic efficacy, patients treated with cDDP suffer from severe side effects and, very often, tumors become resistant to the drug. Aiming to develop more efficient and less toxic agents, which display a broader spectrum of anticancer activity, thousands of new metal-based compounds have been synthesized and tested. These include mono- and polynuclear chelates of platinum (II), palladium (II), and other transition metal ions. ${ }^{1-21}$ In this context, the prior understanding of the biochemical mechanisms underlying the structure-activity relationships ruling cDDP activity is fundamental for an effective and rational design of third-generation anticancer drugs.

Vibrational spectroscopy [infrared, Raman and, more recently, inelastic neutron scattering (INS)] has proven to be one of the most powerful techniques for molecular structure studies. On the other hand, quantum inorganic chemistry has become a general complementary tool that provides a deeper understanding of the structures and corresponding spectra of the systems under study. The usefulness of theoretical calculations to assist vibrational spectroscopy studies has been recently highlighted. ${ }^{22,23}$ With the huge number of available theory levels [all-electron (AE) basis sets and effective core potentials (ECP's)] and theoretical approaches [Hartree-Fock (HF), density functional theory (DFT), and Møller-Plesset (MP), among others], it becomes of particular relevance to perform a systematic evaluation of the successes and failures of each one, in order to select the best method to be used in the study of Pt(II) and other transition metal complexes. In

\footnotetext{
a) Author to whom correspondence should be addressed. Tel./FAX: +351239-826541. Electronic mail: ama1@ portugalmail.pt
}

other words, it is important to determine the theoretical method yielding the best accuracy versus computational cost ratio. As the complexity of the system increases (namely, when studying polynuclear Pt complexes) it is expected that the $\mathrm{AE}$ basis sets rapidly become prohibitively expensive in computational terms. Therefore, it is particularly meaningful to find alternatives (e.g., ECP combinations) capable of yielding comparable accuracies.

To the best of our knowledge, only three reported works compare the efficacy of different theory levels in the study of cDDP. $^{24-26}$ In a first one by Pavankumar et al. ${ }^{25}$ the accuracies of different AE basis sets, at the HF, MP2, MP3, and MP4 protocols, were evaluated and compared in the light of some experimental structural parameters and vibrational frequencies. More recently, Wysokiński and Michalska ${ }^{24,26}$ examined the efficacy of different DFT methods, at the ECP theory level, in describing the molecular structure of cisplatin. These authors concluded that the mPW1PW DFT protocol is remarkably superior to the widely used B3LYP. ${ }^{26}$ However, only incomplete vibrational assignments of cDDP spectra are to be found in the literature. In fact, none of the reported studies assigns the low-frequency vibrational modes, namely, the $\mathrm{NH}_{3}$ torsion and the skeletal deformation modes.

In the present study, different theory methods (HF, DFT, and MP2) combined with different AE basis sets, for describing the nonmetal atoms, are used to predict the molecular structure and vibrational spectra of cDDP. The effectiveness of each theoretical level is evaluated on the basis of the best compromise between accuracy and computational demands. The accuracy of the calculated results is determined via comparison of the calculated values with the experimental ones, namely, the $x$-ray structural data found in the literature ${ }^{27}$ and the recorded vibrational spectra [Raman, Fourer transform 
infrared (FTIR), and INS]. The same type of evaluation, regarding different ECP combinations, will be presented in a future publication.

\section{EXPERIMENTAL}

\section{A. Computational details}

All the calculations were performed on a PC cr, using the GAUSSIAN 03W (G03W) package. ${ }^{28}$

Geometries were fully optimized by the Berny algorithm, using redundant internal coordinates, within symmetry constraints. The optimization convergence criteria for the cut offs of forces and step sizes considered were 0.000015 hartree/bohr for maximum force, 0.000010 hartree/bohr for root-mean-square force, $0.000060 \mathrm{bohr}$ for maximum displacement, and 0.000040 bohr for root-mean-square displacement $(\mathrm{G} 03 \mathrm{~W}$ keyword opt $=$ tight $)$. In all cases, vibrational frequency calculations were performed, at the same theory level, in order to verify that the geometries correspond to a real minimum in the potential energy surface (no negative eigenvalues) as well as to quantify the zero-point vibrational energy correction (zpve).

For the nonmetal atoms, different $\mathrm{AE}$ basis sets, as defined in $\mathrm{G} 03 \mathrm{~W}$, were tested: $6-31 \mathrm{G}(d), 6-31 \mathrm{G}(d, p)$, 6-31G $(3 d f, 2 p d), 6-311 \mathrm{G}(d), 6-311 \mathrm{G}(d, p), 6-311 \mathrm{G}(3 d f$, $2 p d)$, and $6-311++\mathrm{G}(3 d f, 2 p d)$. In all cases the relativistic pseudopotentials developed by Hay and Wadt, ${ }^{29}$ in a doublezeta splitting scheme, were used, as implemented in G03W (keyword LANL2DZ), to describe the platinum atom. This Pt-ECP scheme considers the outermost core electrons $\left(5 s^{2}\right.$ and $5 p^{6}$ ) explicitly in the valence shell. Inclusion of a polarization function at the $\mathrm{Pt}$ atom, by augmenting the valence shell with an $f$-function $\left(\xi_{\mathrm{Pt}}=0.993\right)^{30}$, was also tested.

In order to evaluate the effect of electron correlation (known to be particularly meaningful in systems containing transition metal atoms ${ }^{31}$ ) on the predicted structural parameters and vibrational frequencies, different theoretical methods were considered. Thus, all the AE basis sets were tested at the HF theory level and with two different DFT approaches: (i) the widely used B3LYP, which includes a mixture of HF and DFT exchange terms and the gradientcorrected functionals of Lee, Yang, and Parr, as proposed and parametrized by Becke, ${ }^{32,33}$ and (ii) the mPW1PW method, which comprises a modified version of the exchange term of Perdew-Wang and the Perdew-Wang 91 correlation functional. ${ }^{34,35}$ For each DFT method, the effect of two types of integration grids was considered: (a) a pruned grid of 75 radial shells and 302 angular points per shell (G03W keyword grid $=75$ 302, specifying a FineGrid), and (b) a pruned grid of 99 radial shells and 590 angular points per shell (G03W keyword grid=99 590, defining an UltraFineGrid).

Furthermore, the second-order Møller-Plesset perturbation theory (MP2) was tested, with the smaller AE basis sets 6-31G $(d), 6-31 \mathrm{G}(d, p)$, and 6-31G $(d)$, and 6-31G $(d, p)$. The obtained values were used as an additional reference for evaluating the quality of the results yielded by the other protocols (HF, B3LYP, and mPW1PW).
TABLE I. List of theoretical levels considered in this study for the $\mathrm{Pt}$ ligands, as well as the corresponding nomenclature used throughout the text and tables.

\begin{tabular}{lclc}
\hline \hline Theoretical level & Nomenclature & AE basis set & \\
\hline Hartree-Fock & HF & $6-31 \mathrm{G}(d)$ & Nomenclature \\
MP2 & MP2 & $6-31 \mathrm{G}(d, p)$ & AE1 \\
& & $6-31 \mathrm{G}(3 d f, 2 p d)$ & AE2 \\
B3LYP & B3L & $6-311 \mathrm{G}(d)$ & AE3 \\
mPW1PW & mPW1 & $6-311 \mathrm{G}(d, p)$ & AE4 \\
& & $6-311 \mathrm{G}(3 d f, 2 p d)$ & AE5 \\
& & $6-311 \mathrm{G}++(3 d f, 2 p d)$ & AE6 \\
\end{tabular}

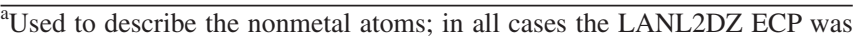
used to describe the metal atom.

The different theory methods and basis set combinations are collected in Table I along with the corresponding nomenclature used throughout the discussion.

Due to the allowed free rotation of the ammine groups, three geometries - two with $C_{2 v}$ symmetry and one with $C_{s}$ symmetry-were found (Fig. 1). All attempts made to achieve other geometries (namely, geometries displaying all ammine hydrogen atoms lying out of the $\mathrm{Cl}-\mathrm{Pt}-\mathrm{Cl}$ plane) failed, as all converged to one of the conformations represented in Fig. 1.

Evaluation of the theoretical level accuracy. As previously stated, one of the aims of the present work is to find a theoretical method that yields the best theoretical description of the experimental data available for cDDP, at the lowest computational cost possible. The first test for accuracy was based on the prediction of the structural parameters of the system. Two main criteria were used: (i) individual deviation $\left(\Delta_{i}\right)$ of each calculated structural parameter characterizing the heavy atoms (bond lengths and angles) in relation to the corresponding experimental values $\left(\Delta_{i}=\right.$ theoretical value - experimental value), and (ii) overall mean percent difference determined as

$$
\Delta \Delta=\frac{\sum_{i=1}^{n}\left(\left|\Delta_{i}\right| / x_{i}\right)}{n} \times 100
$$

where $n=$ number of structural parameters considered and $x_{i}=$ experimental value of a given structural parameter (from the reported $\mathrm{x}$-ray data ${ }^{27}$ ).

The performance of the different theoretical levels was then further evaluated by verifying their ability to predict the vibrational frequencies of cisplatin. This was carried out in the light of a balance between two main factors: highest accuracy [lowest root-mean-square (rms) deviation] and lowest central processor unit time (CPUT).
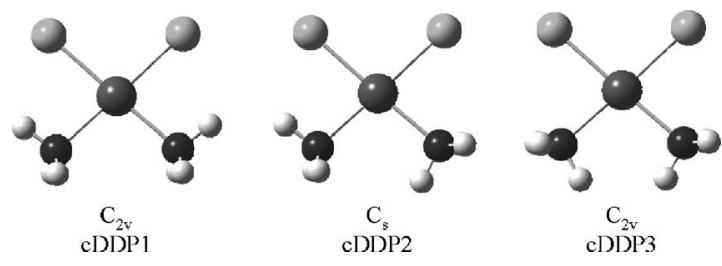

FIG. 1. Optimized geometries for cDDP. 
In general, the calculated vibrational frequencies are found to be overestimated as compared to the experimental ones. Therefore, the theoretical values are usually scaled by carefully chosen scaling factors. In the present work, three different models have been considered for determining the most appropriate scaling factor for the system studied.

(1) Use of a single scaling factor $(\lambda)$ for all frequencies, determined as

$\lambda=\frac{\sum_{i=1}^{n}\left(\nu_{i} / \omega_{i}\right)}{n}$,

where $\nu_{i}$ and $\omega_{i}$ represent the $i$ th experimental and theoretical frequencies, respectively, and $n$ is the number of vibrational frequencies to be compared.

(2) Use of two different scaling factors $\left(\lambda_{1}\right.$ and $\left.\lambda_{2}\right)$, one for the wavenumbers lying below $400 \mathrm{~cm}^{-1}$ and another for those above $400 \mathrm{~cm}^{-1}$, as suggested by Scott and Radom. ${ }^{36}$ The two scaling factors were determined in accordance to Eq. (2).

(3) Use of different scaling factors for different frequency sets $\left(\lambda_{s}\right)$. In this approach, an individual scaling factor was first determined as

$\lambda_{i}=\frac{\nu_{i}}{\omega_{i}}$,

and the different frequencies were grouped by the principle of similar $\lambda_{i}$-values. The sets of scaling factors were determined as

$\lambda_{s}=\frac{\sum_{i=1}^{n_{g}} \lambda_{i}}{n_{s}}$,

where $n_{s}$ stands for the number of frequencies in a particular frequency set. The effect of considering three and four different sets of vibrational frequencies (i.e., three and four different $\lambda_{s}$ ) was assessed.

Finally, for each approach, the resulting individual and overall $\mathrm{rms}$ deviations were determined in accordance to the following:

$$
\begin{aligned}
& \Delta_{i}=\frac{\sqrt{\left(\nu_{i}-\lambda \omega_{i}\right)^{2}}}{\nu_{i}}, \\
& \mathrm{rms}=\frac{\sum_{i=1}^{n} \Delta_{i}}{n},
\end{aligned}
$$

where $\lambda$ stands for $\lambda_{i}$ or $\lambda_{s}$, respectively.

\section{B. Vibrational details}

cDDP (99.99\%) was purchased from Sigma-Aldrich (Sintra, Portugal).

Room-temperature Fourier transform Raman (FTRaman) spectra were recorded on an RFS-100 Bruker FT spectrometer, using a Nd:YAG (yttrium aluminum garnet) laser with an excitation wavelength of $1064 \mathrm{~nm}$. Each spectrum is the average of three repeated measurements of 150 scans, at $2 \mathrm{~cm}^{-1}$ resolution.
TABLE II. Calculated relative energies $\left(\mathrm{kJ} \mathrm{mol}^{-1}\right)$ for the three conformations of cDDP (Fig. 1), using the all-electron basis sets at the nonmetal atoms and LANL2DZ, augmented with an $f$-polarization function at the valence shell, at the Pt atom. The calculated values after zpve correction are presented in parentheses when the conformation was found to be a real minimum in the potential energy surface. In the case of the DFT results, the values are those obtained by using the FineGrid option.

\begin{tabular}{cccc}
\hline \hline Theoretical level & cDDP1 & cDDP2 & cDDP3 \\
\hline HF-AE1 & $0.00(0.00)$ & $0.53(0.38)$ & 1.58 \\
HF-AE2 & 0.00 & 0.74 & 1.99 \\
HF-AE3 & 0.00 & 0.63 & 1.71 \\
HF-AE4 & $0.45(0.00)$ & $0.00(0.12)$ & $0.06(0.34)$ \\
HF-AE5 & $0.27(0.00)$ & $0.00(0.25)$ & $0.20(0.65)$ \\
HF-AE6 & $0.00(0.00)$ & $0.36(0.35)$ & 1.32 \\
HF-AE7 & $0.00(0.00)$ & $0.56(0.62)$ & 1.72 \\
B3L-AE1 & 0.00 & 3.15 & 6.61 \\
B3L-AE2 & 0.00 & 3.50 & 7.30 \\
B3L-AE3 & 0.00 & 3.28 & 6.86 \\
B3L-AE4 & 0.00 & 1.79 & 3.88 \\
B3L-AE5 & 0.00 & 2.14 & 4.59 \\
B3L-AE6 & 0.00 & 2.81 & 6.08 \\
B3L-AE7 & 0.00 & 3.06 & 6.60 \\
mPW1-AE1 & 0.00 & 2.93 & 6.25 \\
mPW1-AE2 & 0.00 & 3.40 & 7.18 \\
mPW1-AE3 & 0.00 & 3.45 & 7.25 \\
mPW1-AE4 & 0.00 & 1.61 & 3.60 \\
mPW1-AE5 & 0.00 & 2.16 & 4.69 \\
mPW1-AE6 & 0.00 & 3.00 & 6.55 \\
mPW1-AE7 & 0.00 & 3.27 & 7.09 \\
MP2-AE1 & 0.00 & 1.02 & 2.22 \\
MP2-AE2 & 0.00 & 1.17 & 2.43 \\
MP2-AE4 & $1.16(0.00)$ & $0.50(0.17)$ & $0.00(0.44)$ \\
MP2-AE5 & $0.24(0.00)$ & $0.00(0.63)$ & $0.06(1.35)$ \\
\hline \hline
\end{tabular}

The FTIR spectra at room temperature were recorded over the $400-4000 \mathrm{~cm}^{-1}$ region on a Mattson 7000 FTIR spectrometer, using a globar source, a deuterated triglycine sulphate detector, and potassium bromide cells. Each spectrum was composed of $32 \mathrm{scans}$, with $2 \mathrm{~cm}^{-1}$, resolution and triangular apodization.

\section{RESULTS AND DISCUSSION}

\section{A. Conformational analysis}

Table II lists the relative energies (uncorrected for zpve) calculated for the three optimized geometries found for cDDP, at the different AE theory levels (with LANL2DZ augmented with an $f$-polarization function at the $\mathrm{Pt}$ atom). Whenever more than one minimum (no negative eigenvalue) is obtained, the relative energies after zpve correction are included (in parentheses). The corresponding values calculated without a polarization function at the metal atom, either considering the FineGrid or the UltraFineGrid options, are presented in Tables SII and SIIa (supplementary material), ${ }^{37}$ respectively.

Analysis of the data comprised in Table II shows that the number of minima as well as their relative conformational energies strongly depend on the theory level used. Indepen- 
TABLE III. Calculated structural parameters (distances in picometers and angles in degrees) for the lowest-energy minimum predicted for cDDP (geometry cDDP1), using the all-electron basis sets at the nonmetal atoms and LANL2DZ, augmented with an $f$-polarization function at the valence shell, at the Pt atom. In the case of the DFT results, the values are those obtained by using the FineGrid option.

\begin{tabular}{|c|c|c|c|c|c|c|c|c|c|c|c|}
\hline \multirow{2}{*}{$\begin{array}{c}\text { Theoretical level } \\
\text { HF-AE1 }\end{array}$} & \multicolumn{2}{|c|}{$\mathrm{Pt}-\mathrm{Cl}^{\mathrm{a}}$} & \multicolumn{2}{|c|}{$\mathrm{Pt}-\mathrm{N}^{\mathrm{a}}$} & \multicolumn{2}{|c|}{$\mathrm{Cl}-\mathrm{Pt}-\mathrm{Cl}^{\mathrm{a}}$} & \multicolumn{2}{|c|}{$\mathrm{Cl}-\mathrm{Pt}-\mathrm{N}^{\mathrm{a}}$} & \multicolumn{2}{|c|}{$\mathrm{N}-\mathrm{Pt}-\mathrm{N}^{\mathrm{a}}$} & \multirow{2}{*}{$\frac{\Delta \Delta^{\mathrm{b}}}{5.3}$} \\
\hline & 235.5 & 2.4 & 211.1 & 11.1 & 95.7 & 3.8 & 84.6 & -5.7 & 95.2 & 8.2 & \\
\hline HF-AE2 & 235.5 & 2.4 & 211.3 & 11.3 & 95.8 & 3.9 & 84.4 & -5.9 & 95.3 & 8.3 & 5.4 \\
\hline HF-AE3 & 232.2 & -0.9 & 211.0 & 11.0 & 96.0 & 4.1 & 84.3 & -6.0 & 95.4 & 8.4 & 5.3 \\
\hline HF-AE4 & 235.9 & 2.8 & 210.7 & 10.7 & 95.2 & 3.3 & 85.0 & -5.3 & 94.8 & 7.8 & 5.0 \\
\hline HF-AE5 & 235.9 & 2.8 & 211.0 & 11.0 & 95.4 & 3.5 & 84.8 & -5.5 & 95.1 & 8.1 & 5.2 \\
\hline HF-AE6 & 232.5 & -0.6 & 210.8 & 10.8 & 95.6 & 3.7 & 84.5 & -5.8 & 95.3 & 8.3 & 5.1 \\
\hline HF-AE7 & 232.5 & -0.6 & 210.5 & 10.5 & 95.6 & 3.7 & 84.4 & -5.9 & 95.5 & 8.5 & 5.2 \\
\hline B3L-AE1 & 233.8 & 0.7 & 210.4 & 10.4 & 95.2 & 3.3 & 83.3 & -7.0 & 98.1 & 11.1 & 5.9 \\
\hline B3L-AE2 & 233.9 & 0.8 & 210.5 & 10.5 & 95.5 & 3.6 & 83.1 & -7.2 & 98.4 & 11.4 & 6.1 \\
\hline B3L-AE3 & 230.6 & -2.5 & 210.3 & 10.3 & 95.7 & 3.8 & 82.9 & -7.4 & 98.5 & 11.5 & 6.4 \\
\hline B3L-AE4 & 234.4 & 1.3 & 210.1 & 10.1 & 94.6 & 2.7 & 83.9 & -6.4 & 97.7 & 10.7 & 5.6 \\
\hline B3L-AE5 & 234.5 & 1.4 & 210.5 & 10.5 & 94.9 & 3.0 & 83.5 & -6.8 & 98.1 & 11.1 & 5.9 \\
\hline B3L-AE6 & 231.2 & -1.9 & 209.9 & 9.9 & 95.5 & 3.6 & 83.1 & -7.2 & 98.3 & 11.3 & 6.1 \\
\hline B3L-AE7 & 231.2 & -1.9 & 209.5 & 9.5 & 95.5 & 3.6 & 83.0 & -7.3 & 98.4 & 11.4 & 6.1 \\
\hline mPW1-AE1 & 231.3 & -1.8 & 207.6 & 7.6 & 94.9 & 3.0 & 83.5 & -6.8 & 98.0 & 11.0 & 5.6 \\
\hline mPW1-AE2 & 231.4 & -1.7 & 207.7 & 7.7 & 95.2 & 3.3 & 83.2 & -7.1 & 98.3 & 11.3 & 5.8 \\
\hline mPW1-AE3 & 228.1 & -5.0 & 207.4 & 7.4 & 95.7 & 3.8 & 82.9 & -7.4 & 98.6 & 11.6 & 6.3 \\
\hline mPW1-AE4 & 231.7 & -1.4 & 207.4 & 7.4 & 94.3 & 2.4 & 84.0 & -6.3 & 97.7 & 10.7 & 5.2 \\
\hline mPW1-AE5 & 231.8 & -1.3 & 207.6 & 7.6 & 94.7 & 2.8 & 83.5 & -6.8 & 98.2 & 11.2 & 5.6 \\
\hline mPW1-AE6 & 228.5 & -4.6 & 207.2 & 7.2 & 95.5 & 3.6 & 83.0 & -7.3 & 98.4 & 11.4 & 6.1 \\
\hline mPW1-AE7 & 228.5 & -4.6 & 206.9 & 6.9 & 95.5 & 3.6 & 83.0 & -7.3 & 98.6 & 11.6 & 6.2 \\
\hline MP2-AE1 & 232.2 & -0.9 & 204.9 & 4.9 & 94.5 & 2.6 & 84.2 & -6.1 & 97.1 & 10.1 & 4.8 \\
\hline MP2-AE2 & 232.1 & -1.0 & 204.6 & 4.6 & 94.7 & 2.8 & 83.9 & -6.4 & 97.4 & 10.4 & 5.0 \\
\hline MP2-AE4 & 232.2 & -0.9 & 204.3 & 4.3 & 94.0 & 2.1 & 84.6 & -5.7 & 96.8 & 9.8 & 4.5 \\
\hline MP2-AE5 & 232.0 & -1.1 & 203.7 & 3.7 & 94.7 & 2.8 & 83.9 & -6.4 & 97.6 & 10.6 & 4.9 \\
\hline
\end{tabular}

Difference between calculated and experimental values in boldface; in the case of Pt-Cl, Pt-N, and Cl-Pt-N the average of the two experimental values was used. The experimental individual values are Pt-Cl: 233.3 and $232.8 \mathrm{pm} ; \mathrm{Pt}-\mathrm{N}: 195.0$ and $205.0 \mathrm{pm} ; \mathrm{Cl}-\mathrm{Pt}-\mathrm{N}: 88.5^{\circ}$ and $92.0^{\circ}$; $\mathrm{Cl}-\mathrm{Pt}-\mathrm{Cl}$ : $91.9^{\circ}$; and N-Pt-N: 87.0 $0^{\circ}$ (Ref. 25).

${ }^{\mathrm{b}}$ Overall mean percent difference obtained by Eq. (1).

dently of the basis set, both DFT approaches yield geometry cDDP1 as the single minimum. The type of the basis set used only affects the magnitude of cDDP1 stability relative to the cDDP2 and cDDP3. In the case of the HF and MP2 calculations, in turn, the basis set dependence is also reflected on the number of cisplatin conformers. HF-AE2, HF-AE3, MP2$\mathrm{AE} 1$, and MP2-AE2 theory levels predict CDDP1 as the sole minimum, similarly to the DFT calculations, while splitting of the basis set (HF-AE4, HF-AE5, MP2-AE4, and MP2AE5) leads to three close lying minima. With these theory levels, cDDP1 only becomes the lowest-energy geometry when the zpve correction is taken into account. On the other hand, the HF calculations using the AE1, AE6, and AE7 basis sets point to an intermediate situation: CDDP1 and cDDP2 are real minima (the former predicted to be slightly more stable), and cDDP3 is a saddle point.

These results are not affected by the presence or absence of an $f$-polarization function at the Pt-atom valence shell (Table II versus Table SII), nor by the type of integration grid, in the case of the DFT calculations (Table II versus Table SIIa). In fact, adding an $f$-polarization function to the platinum valence shell only affects the magnitude of the relative energies, the largest effect being observed for the DFT calculations. Changing the type of integration grid leads to a similar but smoother effect.
On the whole, independently of the basis set considered, the DFT calculations yield larger energy differences between optimized geometries, as compared to HF and MP2 approaches. This probably results from a different weight attributed by the distinct methods (DFT versus HF and MP2) to the $\mathrm{H} \cdots \mathrm{H}$ repulsions and the stabilizing effect of the intramolecular $\mathrm{N}-\mathrm{H} \cdots \mathrm{Cl}$ interactions: the DFT methods giving more relevance to the former effect, while the opposite seems to be the case at the HF and MP2 theory levels.

Finally, it is clear that the number of predicted conformers depends on both the theoretical method and the AE basis set used to describe the nonmetal atoms. This result disagrees from the one reported by Pavankumar et al., ${ }^{25}$ who considered that all three geometries (cDDP1, cDDP2, and cDDP3) correspond to real minima in the cDDP potential energy surface, independently of the theoretical level considered in the calculations.

\section{B. Structural analysis}

Table III compiles some selected structural parameters predicted for cDDP1 geometry of cisplatin (using LANL2DZ augmented with a polarization function at the $\mathrm{Pt}$ atom). The $\Delta$-values for each structural parameter, as well as the $\Delta \Delta$-values [Eq. (1) from the Experimental section] obtained 
are presented in boldface. The results using no polarization function at the Pt atom, considering either the FineGrid or the UltraFineGrid, are shown in Tables SIII and SIIIa of the supplementary material, ${ }^{37}$ respectively.

The values from Table III clearly evidence that the MP2 methodology leads to the best overall agreement (smallest $\Delta \Delta$-value, in all cases $\leqslant 5.0 \%$ ), while the largest $\Delta \Delta$-values are obtained with the B3LYP protocol (in all cases $\geqslant 5.6 \%$ ). $\mathrm{mPW} 1 \mathrm{PW}$ and HF methodologies yield an intermediate accuracy, the latter giving rise to smaller $\Delta \Delta$-values. The results are also basis set dependent, with AE4 yielding the highest overall accuracy (lowest $\Delta \Delta$-value) within all four methodologies (HF, B3LYP, mPW1PW, and MP2).

Comparison of Tables III and SIII shows that considering an $f$-polarization function at the metal leads only to a slight improvement of the overall accuracy (lowering of the $\Delta \Delta$-values). The improvement is more pronounced at the MP2 formalism at all theory levels. In contrast, no enhancement of the overall performance is observed by considering the more computationally demanding integration grid (Table SIIIa versus Table SIII). In fact, the use of the UltraFineGrid option does not affect the description of any of the structural parameters of cDDP.

At this point, it is relevant to assess the accuracy of the different theoretical levels for describing each structural parameter independently, since this type of analysis may lead to several noteworthy observations.

Bond distances. Regarding the $\mathrm{Pt}-\mathrm{Cl}$ bond length, both the HF and B3LYP methods either overestimate or underestimate this structural parameter, depending on the basis set used. AE1, AE2, AE4, and AE5 yield overestimated Pt-Cl distances, while AE3, AE6, and AE7 give underestimated values. In contrast, mPW1PW and MP2 calculations predict underestimated values, independently of the basis set used to describe the nonmetal heavy atom.

At the MP2 formalism all basis sets give an identical description of the $\mathrm{Pt}-\mathrm{Cl}$ distance, with $\Delta$-values not exceeding $1.1 \mathrm{pm}$. For the HF calculations, the best agreement is achieved when the $(3 d f, 2 p d)$-polarization function scheme is considered (AE3, AE6, and AE7). Splitting of the valence shell of the nonmetal atoms (AE3 $\rightarrow \mathrm{AE} 6)$ is responsible for a very slight improvement of the $\mathrm{Pt}-\mathrm{Cl}$ distance $(\Delta=-0.9$ $\rightarrow \Delta=-0.6)$. On the other hand, addition of a diffuse function on the nonmetal heavy atoms (AE6 $\rightarrow$ AE7) has no effect on this parameter.

Among the DFT protocols, the best $\mathrm{Pt}-\mathrm{Cl}$ description is achieved with B3L-AE1 and B3L-AE2, and mPW1-AE4 and mPW1-AE5. This suggests that at the DFT theory level the use of the $(3 d f, 2 p d)$-polarization function scheme leads to a (considerably) worsening of the theoretical description of the $\mathrm{Pt}-\mathrm{Cl}$ bond.

Considering the effect of using a polarization function at the metal (Table III versus Table SIII), it is noteworthy that the largest effect is observed within the MP2 approach. In fact, at both DFT methodologies, the improvements detected are not larger than $1 \mathrm{pm}$, independently of the basis set considered at the nonmetal atoms. Similar improvements are observed with the HF methodology, for all AE basis sets.

Within the MP2 protocol, in turn, the $\mathrm{Pt}-\mathrm{Cl}$ distance is significantly decreased (by $2.5 \mathrm{pm}$ ), going from overestimated to underestimated values (Table SIII $\rightarrow$ Table III). On the whole, it can be concluded that the use of polarization functions to describe the metal valence shell leads to a refinement of 0.3-0.7 pm, depending on the AE basis set used.

Concerning the description of the $\mathrm{Pt}-\mathrm{N}$ distance, it is evident that the mPW1PW methodology yields clearly better results than $\mathrm{HF}$ and B3LYP, at all AE levels. As expected, the lowest deviation from experimental values is observed for the MP2 calculations. On the other hand, the $\mathrm{Pt}-\mathrm{N}$ distance is not as basis set dependent as the $\mathrm{Pt}-\mathrm{Cl}$ parameter, as all theory levels give rise to overestimated $\mathrm{Pt}-\mathrm{N}$ distances. In addition, the deviations observed for each of the theoretical formalisms considered (HF, B3LYP, mPW1PW and MP2) are within a relatively narrow range (HF: $10.5-11.3 \mathrm{pm}$; B3LYP: 9.5-10.5 pm; mPW1PW: 6.9-7.7 pm; MP2: $3.7-4.9 \mathrm{pm})$.

Finally, considering the effect of including a polarization function at the metal (Table III versus Table SIII), the improvements observed are larger than the ones verified for the $\mathrm{Pt}-\mathrm{Cl}$ bond length, at all theory levels. As for the Pt-Cl distance the largest improvements are observed with the MP2 approach (values ranging from -3.3 to $-3.8 \mathrm{pm}$ ). Actually, inclusion of an $f$-polarization function at the Pt valence shell is a requirement to render MP2 calculations significantly more accurate than the HF and mPW1PW methodologies for representing this structural parameter.

Bond angles. Regarding the bond angles, the four protocols yield overestimated $\mathrm{Cl}-\mathrm{Pt}-\mathrm{Cl}$ and $\mathrm{N}-\mathrm{Pt}-\mathrm{N}$ angles, and underestimated $\mathrm{Cl}-\mathrm{Pt}-\mathrm{N}$ values, independently of the AE basis used. The best theoretical-experimental agreement for the $\mathrm{Cl}-\mathrm{Pt}-\mathrm{Cl}$ bond angle is obtained within the MP2 formalism. The two bond angles involving the nitrogen atom $(\mathrm{N}-\mathrm{Pt}-\mathrm{N}$ and $\mathrm{Cl}-\mathrm{Pt}-\mathrm{N}$ ), on the other hand, are clearly better reproduced by the HF protocol. The DFT calculations yield lower accuracy than HF and MP2 in the description of both the $\mathrm{N}-\mathrm{Pt}-\mathrm{N}$ and $\mathrm{Cl}-\mathrm{Pt}-\mathrm{N}$ bonds. However, both B3LYP and mPW1PW lead to slightly more accurate $\mathrm{Cl}-\mathrm{Pt}-\mathrm{Cl}$ angles than HF. Finally, comparing the performance of both DFT methods, mPW1PW yields clearly better results than B3LYP.

The effect of considering a polarization function to describe the metal center is much smoother than that observed for the bond distances (Table III versus Table SIII). The largest effects are observed for the MP2 protocol, where improvements up to $0.4^{\circ}$ are obtained, as opposed to $\Delta \leqslant 0.2^{\circ}$ for the other theoretical protocols (HF, B3LYP, and mPW1PW).

The calculated structural parameters at the HF-AE1, HFAE2, HF-AE4, HF-AE5, MP2-AE1, and MP2-AE4 levels are, naturally, very similar to the results obtained by Pavankumar et al., ${ }^{25}$ using the same theoretical level (theoretical method/AE/ECP). The small differences presently found are probably due to the use of tighter convergence criteria than the one in the calculations of Pavankumar et al. Comparison with the results by Wysokiński and Michalska, ${ }^{26}$ in turn, shows that the herein calculated values are significantly more accurate, at all theoretical levels considered. In fact, the use of Eq. (1) for the data predicted by Wysokiński and Michalska (which considered ECP's to describe all atoms) yields 
TABLE IV. Central processor unit time (CPUT) required for a vibrational frequency calculation using the all-electron basis sets at the nonmetal atoms and LANL2DZ at the Pt atom. NO and YES stand, respectively, for without and with an $f$-polarization function at the Pt-valence shell (using the FineGrid option in the DFT calculations); UFG represents the UltraFineGrid option, in the DFT calculations (with no polarization function at the Pt-valence shell).

\begin{tabular}{|c|c|c|c|c|c|c|c|}
\hline \multirow[b]{2}{*}{ Theoretical level } & \multicolumn{3}{|c|}{ CPUT $^{\mathrm{a}}$} & \multirow[b]{2}{*}{ Theoretical level } & \multicolumn{3}{|c|}{ CPUT $^{\mathrm{a}}$} \\
\hline & NO & YES & UFG & & NO & YES & UFG \\
\hline HF-AE2 & 2.8 & 4.5 & $\ldots$ & mPW1AE1 & 15.0 & 18.5 & 36.0 \\
\hline HF-AE2 & 2.3 & 6.0 & $\cdots$ & mPW1AE2 & 18.4 & 22.5 & 42.3 \\
\hline HF-AE3 & 54.4 & 65.2 & $\ldots$ & mPW1AE3 & 88.0 & 99.7 & 169.8 \\
\hline HF-AE4 & 2.8 & 7.8 & $\cdots$ & mPW1AE4 & 19.7 & 24.2 & 44.9 \\
\hline HF-AE5 & 3.7 & 10.7 & $\cdots$ & mPW1AE5 & 24.5 & 30.2 & 55.7 \\
\hline HF-AE6 & 79.3 & 92.2 & $\ldots$ & mPW1AE6 & 110.6 & 125.7 & 207.3 \\
\hline HF-AE7 & 122.2 & 131.8 & $\ldots$ & mPW1AE7 & 145.7 & 161.6 & 270.6 \\
\hline B3L-AE1 & 14.8 & 18.1 & 35.3 & MP2AE1 & 22.0 & 28.6 & $\cdots$ \\
\hline B3L-AE2 & 18.0 & 22.2 & 41.5 & MP2AE2 & 40.9 & 50.9 & $\ldots$ \\
\hline B3L-AE3 & 86.7 & 97.3 & 167.0 & & & & \\
\hline B3L-AE4 & 19.4 & 23.7 & 44.2 & MP2AE4 & 59.4 & 70.4 & $\ldots$ \\
\hline B3L-AE5 & 24.1 & 29.6 & 54.7 & MP2AE5 & 93.1 & 113.6 & $\ldots$ \\
\hline B3L-AE6 & 109.8 & 122.4 & 204.8 & & & & \\
\hline B3L-AE7 & 142.6 & 159.1 & 263.8 & & & & \\
\hline
\end{tabular}

${ }^{\mathrm{a} C o m p u t a t i o n a l ~ t i m e ~ r e q u i r e d ~ f o r ~ a ~ f r e q u e n c y ~ c a l c u l a t i o n, ~ i n ~ m i n u t e s . ~}$

$\Delta \Delta$-values of $6.5 \%$ at the HF level, $6.8 \%$ at the B3LYP level, and $6.3 \%$ at the mPW1PW and MP2 levels, quite higher than the values obtained with the present results. As to the prediction of each structural parameter separately, a better reproduction of all structural parameters but one is achieved in the present work. The only exception is the $\mathrm{Pt}-\mathrm{N}$ distance, which are presently more overestimated at the HF and DFT levels than in the work of Wysokiński and Michalska.

In short, independently of the theoretical method (HF, MP2, or DFT), the best overall accuracy, from a structural point of view, is achieved by using the AE4 basis set to describe the nonmetal atoms. Comparing the results obtained with the four AE basis sets tested within all four theoretical methods (namely, AE1, AE2, AE4, and AE5), it is found that MP2 yields the most rigorous results. However, this theoretical method becomes prohibitive as the system complexity increases, which urges for the use of an alternative theoretical method.

The results from Table III suggest that the best choice is the HF-AE4 theoretical level. However, the mPW1-AE4 level is a good alternative since it yields an overall mean percent difference similar to the one obtained at the HF-AE4 theory level (5.2 vs 5.0, respectively). This finding is particularly significant as the results using the mPW1PW protocol are less sensitive to the use of polarization functions at the metal than all the remaining methodologies tested (Table III versus Table SIII). Finally, it is to be noted that mPW1-AE1 constitutes a good alternative when computational efforts are to be reduced further (e.g., when multinuclear platinum complexes are to be studied).

\section{Vibrational analysis}

Table IV lists the CPUT (in minutes) required for a vibrational frequency calculation, for each of the different theoretical levels considered. It is not surprising that an improvement of the nonmetal basis set leads to a considerable increase of CPUT. Inclusion of polarization functions at the
$\mathrm{Pt}$ atom further increases this computational time. Nevertheless, the most pronounced CPUT increase occurs when the UltraFineGrid option is used in the DFT calculations.

These results reinforce the importance of a careful choice of the theoretical level to be used, particularly when large molecular systems are to be analyzed. A satisfactory balance between accuracy and computational demands must be attained. In this context, it is evident that the use of the UltraFineGrid option in the DFT calculations can be ruled out, as it does not lead to a significant improvement of the structural description of cDDP while requiring a considerable increase in CPUT.

Figure 2 shows the experimental FT-Raman spectra recorded for solid cDDP, in the 75-1800 and 3000-3600 cm spectral regions. Some regions are expanded in order to allow the visualization of spectral details otherwise undetectable. The wavenumbers presented are the ones used for the accuracy evaluation of the calculated vibrational frequencies (discussed below). The INS spectra reported by some of us ${ }^{38}$ were considered for the very-low-frequency region.

Cisplatin displays 27 vibrational modes, all Raman active. Considering the most stable calculated conformation (cDDP1), these modes are distributed as $9 \mathrm{a}_{1}+5 \mathrm{a}_{2}+5 \mathrm{~b}_{1}$ $+8 b_{2}$. The low-frequency vibrations (as well as the corresponding nomenclature used throughout the text) are schematically represented in Fig. 3, as they can give rise to some misleading.

The calculated harmonic vibrational frequencies are known to be usually overestimated relative to the experimental wavenumbers, although some modes, namely, the lowfrequency ones, may be significantly underestimated. Thus, a common methodology used consists in scaling the calculated values. While there is a huge volume of work on the determination of scaling factors for organic compounds, ${ }^{36,39-44}$ the same kind of studies on inorganic systems are still missing. Aiming to fulfill this gap, different scaling schemes, varying in the number of frequency sets and vibrational frequency 

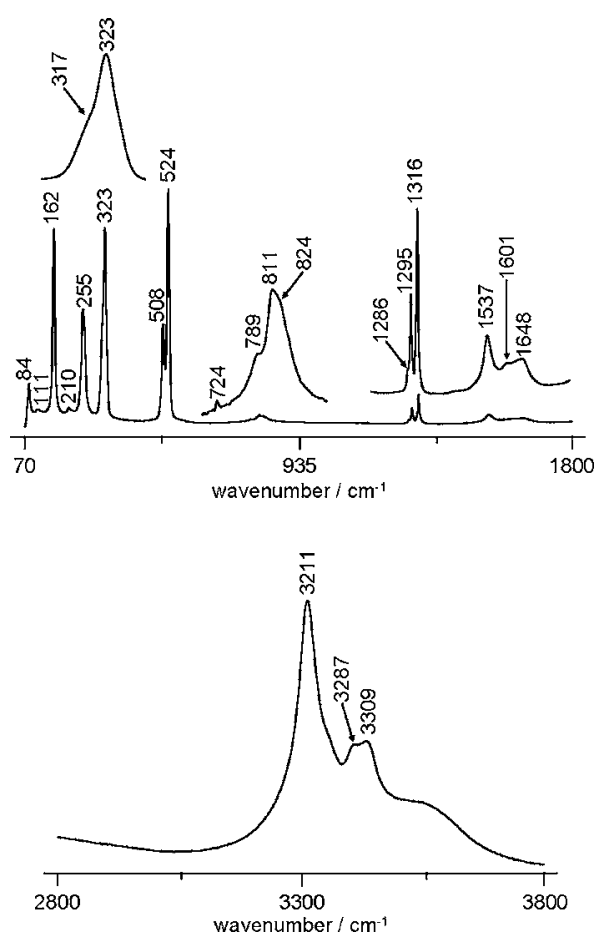

FIG. 2. Room-temperature FT-Raman spectra of solid cDDP, in the 70-1800 and $2800-3800 \mathrm{~cm}^{-1}$ spectral regions. Some regions are expanded in order to allow the visualization of spectral details otherwise undetectable. The wavenumbers indicated are used for the accuracy evaluation of the calculated vibrational frequencies.

grouping, were tested in order to encounter the best scaling factors to be used with a particular theory level.

Some of the results (scaling factors, vibrational modes comprised in each frequency set, and rms values) are comprised in Tables SV-SVg (supplementary material) ${ }^{37}$ Please note that for the three- and four-set grouping schemes (Tables SVd-SVg) only the scaling scheme yielding the lowest rms is presented.
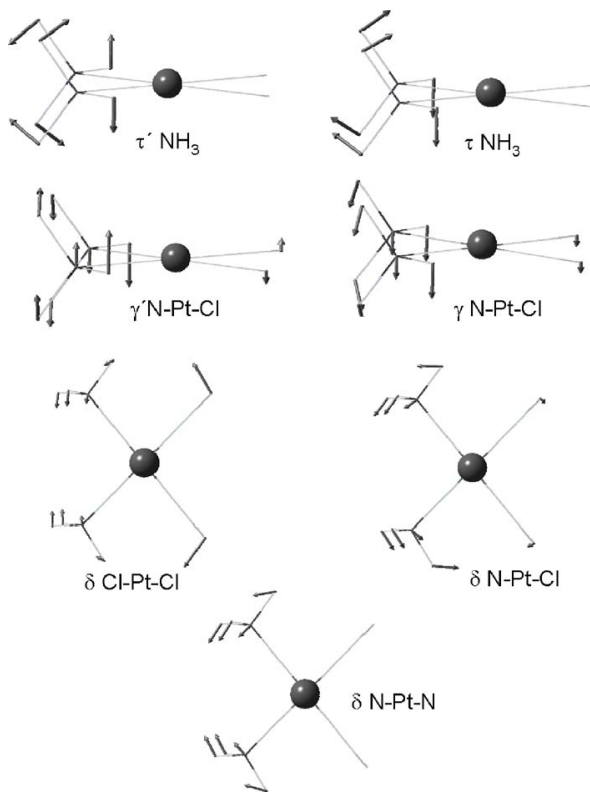

FIG. 3. Schematic representation of the atom motions involved in the lowfrequency vibrational modes of cDDP. The nomenclature used throughout the discussion is indicated.
In all cases, the best theoretical-to-experimental match is achieved with a set of four scaling factors. The use of a single factor, with or without a polarization function at the metal (Tables SV and SVa), gives rise to very high rms values. The use of two scaling factors [one below $\left(\lambda_{1}\right)$ and one above $400 \mathrm{~cm}^{-1}\left(\lambda_{2}\right)$, as suggested by Scott and Radom ${ }^{36}$ ] does not improve the results (Tables SVb and SVc). When three frequency sets are used, a considerable improvement is achieved (Tables SVd and SVe), the most pronounced effects occurring at the HF calculations. Further improvement is gained by considering a four-set frequency grouping scheme (Tables SVf and SVg), while continuing to increase the number of frequency sets (and, consequently, of scaling factors) is verified to become insignificant. The improvements achieved by considering $f$-polarization functions at the metal valence shell are very small $(<0.2 \%)$.

The results suggest that grouping of the vibrational frequencies among the defined frequency sets is strongly dependent on the AE basis set considered. Moreover, independently of the basis set, the ammine stretching and deformation modes $\left(\nu_{\mathrm{NH}_{3}}\right.$ and $\delta_{\mathrm{NH}_{3}}$, respectively), and the skeletal in-plane deformation mode $\delta_{\mathrm{N}-\mathrm{Pt}-\mathrm{Cl}}$ (Fig. 3) are the most overestimated ones (lowest scaling factor). In some cases, the $\mathrm{Pt}-\mathrm{Cl}$ stretching mode $\left(\nu_{\mathrm{Pt}-\mathrm{Cl}}\right)$ is also considerably overestimated (HF with AE3, AE5, and AE6; B3LYP with $\mathrm{AE} 1, \mathrm{AE} 3$, and AE4; mPW1PW with AE1, AE2, and AE4; and MP2 with all AE).

All theoretical levels lead to significant underestimation of the Pt-N stretching $\left(\nu_{\mathrm{Pt}-\mathrm{N}}\right)$ and of the skeletal in-plane deformation mode $\delta_{\mathrm{N}-\mathrm{Pt}-\mathrm{N}}$ (Fig. 3). As to the four ammine rocking modes $\left(\rho_{\mathrm{NH}_{3}}\right)$ and to the third in-plane skeletal deformation mode $\delta_{\mathrm{Cl}-\mathrm{Pt}-\mathrm{Cl}}$ (Fig. 3), they are quite accurately predicted by all the theoretical methods. In some cases, despite the four-set scaling scheme, only three scaling factors are required as a $\lambda$-value of 1.0 corresponds to no scaling.

Comparison of Tables SVf and SVg shows that the use of $f$-polarization functions to describe the metal atom does not significantly affect the rms values. Particularly meaningful is the fact that the smallest AE basis set (AE1) yields the same overall deviation from experiment as the more computationally demanding AE4 basis set, with both DFT and MP2 protocols (Table $\mathrm{SVg}$ ). The highest accuracy is achieved at the B3L-AE1 theoretical level $(\mathrm{rms}=1.4 \%)$. The mPW1AE1 theory level yields a higher rms value (1.7\%), but it presents the advantage of grouping related modes in the same frequency set (e.g., the NH3 deformation modes are scaled with the same scaling factor), thus resembling more a traditional frequency grouping. This is particularly significant as transferability of the frequency scaling strategy to other cisplatinlike systems is envisaged.

At this stage it is important to analyze the individual frequency deviations after frequency scaling within the selected theoretical levels (HF-AE4, B3L-AE1, mPW1-AE1, and MP2-AE1) and corresponding scaling models (Table $\mathrm{SVg}$ ). Table $\mathrm{V}$ lists the vibrational frequencies of cDDP, higher than $160 \mathrm{~cm}^{-1}$, after scaling in accordance to Table SVg. The experimental wavenumbers and the individual deviations of the theoretical values from the experimental one are included. 
TABLE V. Calculated harmonic vibrational frequencies for cDDP, after scaling according to the factors contained in Table SVg.

\begin{tabular}{|c|c|c|c|c|c|c|c|c|c|}
\hline \multirow{3}{*}{$\begin{array}{l}\text { Vibrational } \\
\text { mode }^{\mathrm{a}}\end{array}$} & \multicolumn{9}{|c|}{ Theoretical level } \\
\hline & \multicolumn{2}{|c|}{$\mathrm{HF}-\mathrm{AE} 4$} & \multicolumn{2}{|c|}{ B3L-AE1 } & \multicolumn{2}{|c|}{ mPW1-AE1 } & \multicolumn{2}{|c|}{ MP2-AE1 } & \multirow[b]{2}{*}{$\nu^{\mathrm{g}}$} \\
\hline & $\omega^{\mathrm{b}}$ & $\Delta^{\mathrm{c}}$ & $\omega^{\mathrm{d}}$ & $\Delta^{\mathrm{c}}$ & $\omega^{\mathrm{e}}$ & $\Delta^{\mathrm{c}}$ & $\omega^{\mathrm{f}}$ & $\Delta^{\mathrm{c}}$ & \\
\hline$\nu_{\mathrm{as}} \mathrm{NH}_{3}$ & 3338 & 29 & 3354 & 45 & 3330 & 21 & 3329 & 20 & 3309 \\
\hline$\nu_{\mathrm{as}} \mathrm{NH}_{3}$ & 3316 & 29 & 3306 & 19 & 3280 & -7 & 3283 & -4 & 3287 \\
\hline$\nu_{\mathrm{s}} \mathrm{NH}_{3}$ & 3229 & 18 & 3185 & -26 & 3152 & -59 & 3159 & -52 & 3211 \\
\hline$\delta_{\text {as }} \mathrm{NH}_{3}$ & 1625 & -23 & 1608 & -40 & 1635 & -13 & 1636 & -12 & 1648 \\
\hline$\delta_{\mathrm{as}}^{\prime} \mathrm{NH}_{3}$ & 1618 & $-\mathbf{1 0}$ & 1603 & -25 & 1628 & 0 & 1628 & $\mathbf{0}$ & $1628^{h}$ \\
\hline$\delta_{\text {as }} \mathrm{NH}_{3}$ & 1600 & -1 & 1580 & -21 & 1606 & 5 & 1607 & 6 & 1601 \\
\hline$\delta_{\mathrm{as}}^{\prime} \mathrm{NH}_{3}$ & 1592 & 55 & 1573 & 36 & 1598 & 61 & 1599 & 62 & 1537 \\
\hline$\delta_{\mathrm{s}} \mathrm{NH}_{3}$ & 1325 & 9 & 1296 & -20 & 1263 & -53 & 1279 & -37 & 1316 \\
\hline$\delta_{\mathrm{s}}^{\prime} \mathrm{NH}_{3}$ & 1316 & 21 & 1290 & -5 & 1255 & -40 & 1271 & -24 & 1295 \\
\hline$\rho \mathrm{NH}_{3}$ & 839 & 15 & 825 & 1 & 825 & 1 & 830 & 6 & 824 \\
\hline$\rho_{\mathrm{NH}_{3}}^{\prime}$ & 791 & -20 & 791 & -21 & 790 & -21 & 791 & -20 & 811 \\
\hline$\rho \mathrm{NH}_{3}$ & 783 & -6 & 774 & -15 & 779 & -10 & 773 & -16 & 789 \\
\hline$\rho_{\mathrm{NH}_{3}}^{\prime}$ & 756 & 32 & 757 & 33 & 760 & 36 & 753 & 29 & 724 \\
\hline$\nu_{\mathrm{s}} \mathrm{Pt}-\mathrm{N}$ & 515 & -9 & 523 & -1 & 524 & 0 & 519 & -5 & 524 \\
\hline$\nu_{\mathrm{as}} \mathrm{Pt}-\mathrm{N}$ & 502 & -6 & 514 & 6 & 516 & 8 & 515 & 7 & 508 \\
\hline$\nu_{\mathrm{s}} \mathrm{Pt}-\mathrm{Cl}$ & 323 & $\mathbf{0}$ & 331 & 8 & 332 & 9 & 328 & 5 & 323 \\
\hline$\nu_{\mathrm{as}} \mathrm{Pt}-\mathrm{Cl}$ & 312 & -5 & 320 & 3 & 321 & 4 & 321 & 4 & 317 \\
\hline$\delta \mathrm{N}-\mathrm{Pt}-\mathrm{N}$ & 263 & 8 & 253 & -2 & 251 & -4 & 252 & -3 & 255 \\
\hline$\delta \mathrm{N}-\mathrm{Pt}-\mathrm{Cl}$ & 206 & -4 & 208 & -2 & 213 & 3 & 215 & 5 & 210 \\
\hline$\delta \mathrm{Cl}-\mathrm{Pt}-\mathrm{Cl}$ & 160 & -2 & 161 & -1 & 164 & 2 & 160 & -2 & 162 \\
\hline $\mathrm{CPUT}^{\mathrm{i}}$ & \multicolumn{2}{|c|}{2.8} & \multicolumn{2}{|c|}{14.8} & \multicolumn{2}{|c|}{15.0} & \multicolumn{2}{|c|}{22.0} & \\
\hline
\end{tabular}

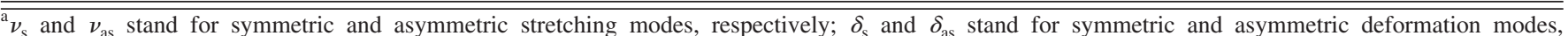
respectively; $\delta=$ skeletal in-plane deformation modes; $\rho$ and $\rho^{\prime}$ stand for in-phase and out-of-phase rocking modes, respectively.

${ }^{\mathrm{b}}$ Theoretical scaled value $\left[\lambda=0.87\left(\nu_{\mathrm{NH}_{3}}\right.\right.$ and $\left.\delta \mathrm{as}_{\mathrm{NH}_{3}}\right), \lambda=0.90\left(\delta \mathrm{s}_{\mathrm{NH}_{3}}\right.$ and $\left.\delta_{\mathrm{N}-\mathrm{Pt}-\mathrm{Cl}}\right), \lambda=0.97\left(\rho_{\mathrm{NH}}, \nu_{\mathrm{Pt}-\mathrm{Cl}}\right.$, and $\left.\delta_{\mathrm{Cl}-\mathrm{Pt}-\mathrm{Cl}}\right)$, and $\lambda=1.14\left(\nu_{\mathrm{Pt}-\mathrm{N}}\right.$ and $\left.\left.\delta_{\mathrm{N}-\mathrm{Pt}}\right)\right]$. ${ }^{\mathrm{c}}$ Deviation from experimental value, after scaling (theoretical-experimental).

${ }^{\mathrm{d}}$ Theoretical scaled value $\left[\lambda=0.94\left(\nu_{\mathrm{NH}_{3}}, \delta \mathrm{as}_{\mathrm{NH}_{3}}\right.\right.$, and $\left.\delta_{\mathrm{N}-\mathrm{Pt}-\mathrm{Cl}}\right), \lambda=0.99\left(\delta \mathrm{s}_{\mathrm{NH}_{3}}\right.$ and $\left.\nu_{\mathrm{Pt}-\mathrm{Cl}}\right), \lambda=1.02\left(\rho_{\mathrm{NH}}\right.$ and $\left.\delta_{\mathrm{Cl}-\mathrm{Pt}-\mathrm{Cl}}\right)$, and $\lambda=1.17\left(\nu_{\mathrm{Pt}-\mathrm{N}}\right.$ and $\left.\left.\delta_{\mathrm{N}-\mathrm{Pt}-\mathrm{N}}\right)\right]$.

${ }^{\mathrm{e}}$ Theoretical scaled value $\left[\lambda=0.92\left(\nu_{\mathrm{NH}_{3}}\right.\right.$ and $\left.\delta_{\mathrm{N}-\mathrm{Pt}-\mathrm{Cl}}\right), \lambda=0.95\left(\delta_{\mathrm{NH}_{3}}\right.$ and $\left.\nu_{\mathrm{Pt}-\mathrm{Cl}}\right), \lambda=1.00\left(\rho_{\mathrm{NH}}\right.$ and $\left.\delta_{\mathrm{Cl}-\mathrm{Pt}-\mathrm{Cl}}\right)$, and $\lambda=1.11\left(\nu_{\mathrm{Pt}-\mathrm{N}}\right.$ and $\left.\left.\delta_{\mathrm{N}-\mathrm{Pt}-\mathrm{N}}\right)\right]$.

${ }^{\mathrm{f}}$ Theoretical scaled value $\left[\lambda=0.92\left(\nu_{\mathrm{NH}_{3}}\right.\right.$ and $\left.\delta_{\mathrm{N}-\mathrm{Pt}-\mathrm{Cl}}\right), \lambda=0.94\left(\delta_{\mathrm{NH}_{3}}\right.$ and $\left.\nu_{\mathrm{Pt}-\mathrm{Cl}}\right), \lambda=0.99\left(\rho_{\mathrm{NH}}{ }_{3}\right.$ and $\left.\delta_{\mathrm{Cl}-\mathrm{Pt}-\mathrm{Cl}}\right)$, and $\lambda=1.10\left(\nu_{\mathrm{Pt}-\mathrm{N}}\right.$ and $\left.\left.\delta_{\mathrm{N}-\mathrm{Pt}-\mathrm{N}}\right)\right]$.

${ }^{\mathrm{g}}$ Experimental FT Raman.

h Observed in the FTIR spectra; probably strongly overlapped with the other $\delta_{\mathrm{NH}_{3}}$ modes in the Raman spectrum.

${ }^{\mathrm{i}} \mathrm{CPUT}$ required for a frequency calculation, in minutes.

At all four theoretical levels, the largest deviations from experiment are observed for the localized $\nu_{\mathrm{NH}_{3}}$ and $\delta_{\mathrm{NH}_{3}}$ modes, probably as a consequence of the intermolecular $\mathrm{N}-\mathrm{H} \cdots \mathrm{Cl}$ interactions. In fact, these close contacts, known to occur in the crystal structure of $\mathrm{cDDP}^{27}$ are not considered in the presently performed quantum mechanical calculations, and are expected to affect more significantly the localized vibrational modes involving the $\mathrm{NH}_{3}$ ligands. The effect of these interactions on the predicted vibrational frequencies of cDDP will be analyzed in a future publication.

In order to make a final choice of the most appropriate theoretical level for accurately representing the conformational, structural, and vibrational properties of cDDP, one final accuracy evaluation must be carried out. The performance of the four theoretical levels (HF-AE4, B3L-AE1, mPW1-AE1, and MP2-AE1) for predicting the very-low vibrational modes - the two ammine torsion modes, $\tau_{\mathrm{NH}_{3}}$ and $\tau^{\prime}{ }_{\mathrm{NH}_{3}}$, and the two skeletal out-of-plane deformation modes, $\gamma_{\mathrm{N}-\mathrm{Pt}-\mathrm{Cl}}$ and $\gamma_{\mathrm{N}-\mathrm{Pt}-\mathrm{Cl}}^{\prime}$ (see Fig. 3) - has to be evaluated.

$\tau_{\mathrm{NH}_{3}}, \tau_{\mathrm{NH}_{3}}^{\prime}, \gamma_{\mathrm{N}-\mathrm{Pt}-\mathrm{Cl}}$, and $\gamma_{\mathrm{N}-\mathrm{Pt}-\mathrm{Cl}}^{\prime}$ modes. Table VI presents the unscaled values calculated for the four lowfrequency modes using the HF-AE1, B3L-AE4, mPW1-AE1, and MP2-AE1 theoretical levels. The calculated individual scaling factors determined for each mode, using Eq. (3), are presented in parentheses.

The main conclusion to be drawn is that the B3L-AE1 and mPW1-AE1 approaches require less frequency scaling sets than both HF-AE4 and MP2-AE1. In the two former cases, the two ammine torsion modes $\left(\tau_{\mathrm{NH}_{3}}\right.$ and $\left.\tau_{\mathrm{NH}_{3}}{ }\right)$ and the out-of-phase skeletal out-of-plane deformation mode $\left(\gamma^{\prime}{ }_{\mathrm{N}-\mathrm{Pt}-\mathrm{Cl}}\right)$ can be grouped in the same frequency set, with $\lambda_{s}=1.54$ (an average of 1.55, 1.50, and 1.56) for B3L-AE1 and $\lambda_{s}=1.52$ (an average of 1.52, 1.51, and 1.54) for mPW1AE1. In contrast, MP2-AE1 requires the definition of two additional frequency sets $\left[\lambda_{s}=1.65\right.$ and 1.99 (an average of 1.98 and 2.00)] for these modes, while HF-AE4 requires three additional frequency sets $\left(\lambda_{s}=1.69,3.37\right.$, and 10.24).

In what concerns the fourth low-lying mode, the results seem to give some advantage to the mPW1-AE1 theoretical level over the B3L-AE1 one. In fact, at the mPW1-AE1 theoretical level the in-phase skeletal out-of-plane deformation mode $\left(\gamma_{\mathrm{N}-\mathrm{Pt}-\mathrm{Cl}}\right)$ can be included in the previously defined third scaling set $\left(\lambda_{s}=1.00\right.$; see Table $\left.\mathrm{SVg}\right)$. In contrast, in the case of the B3L-AE1 results a new scaling set has to be defined with $\lambda_{s}=1.05$. After scaling the four low-frequency 
TABLE VI. Calculated low-frequency vibrational modes (below $160 \mathrm{~cm}^{-1}$ ) for cDDP. The individual scaling factors, determined through Eq. (3), are included in parentheses.

\begin{tabular}{|c|c|c|c|c|c|}
\hline \multicolumn{4}{|c|}{ Theoretical level } & \multirow[b]{2}{*}{ Expt. $^{\mathrm{a}}$} & \multirow[b]{2}{*}{ Vibrational mode } \\
\hline HF-AE4 & B3L-AE1 & mPW1-AE1 & MP2-AE1 & & \\
\hline $\begin{array}{c}152 \\
(1.00)\end{array}$ & $\begin{array}{c}145 \\
(1.05)\end{array}$ & $\begin{array}{c}150 \\
(1.01)\end{array}$ & $\begin{array}{c}148 \\
(1.03)\end{array}$ & 152 & $\gamma_{\mathrm{N}-\mathrm{Pt}-\mathrm{Cl}}$ \\
\hline $\begin{array}{c}125 \\
(1.69)\end{array}$ & $\begin{array}{c}136 \\
(1.55)\end{array}$ & $\begin{array}{c}139 \\
(1.52)\end{array}$ & $\begin{array}{c}128 \\
(1.65)\end{array}$ & 211 & $\tau_{\mathrm{NH}_{3}}^{\prime}$ \\
\hline $\begin{array}{c}60 \\
(3.37)\end{array}$ & $\begin{array}{c}135 \\
(1.50)\end{array}$ & $\begin{array}{c}134 \\
(1.51)\end{array}$ & $\begin{array}{c}102 \\
(1.98)\end{array}$ & 202 & $\tau_{\mathrm{NH}_{3}}$ \\
\hline $\begin{array}{c}17 \\
(10.24)\end{array}$ & $\begin{array}{c}112 \\
(1.56)\end{array}$ & $\begin{array}{c}113 \\
(1.54)\end{array}$ & $\begin{array}{c}87 \\
(2.00)\end{array}$ & 174 & $\gamma_{\mathrm{N}-\mathrm{Pt}-\mathrm{Cl}}^{\prime}$ \\
\hline
\end{tabular}

${ }^{\mathrm{a}}$ Experimental INS vibrational frequencies (Ref. 38).

${ }^{\mathrm{b}} \gamma$ and $\gamma^{\prime}$ stand for in-phase and out-of-phase skeletal out-of-plane deformation modes, respectively, and $\tau$ and $\tau^{\prime}$ stand for in-phase and out-of-phase torsion modes, respectively (see Fig. 3 for mode description).

modes in accordance to the scaling scheme described above, the individual deviations of the calculated frequencies do not exceed $2 \mathrm{~cm}^{-1}$ for the mPW1-AE1 method, while for B3LAE1 the deviations vary between 2 and $6 \mathrm{~cm}^{-1}$ (e.g., a $6 \mathrm{~cm}^{-1}$ shift is obtained for $\tau_{\mathrm{NH}_{3}}: 135 \mathrm{~cm}^{-1} \times 1.54$ $=208 \mathrm{~cm}^{-1}$ ). In other words, mPW1-AE1 not only allows reducing the number of frequency scaling factors but also yields slightly more accurate results as compared to B3LAE1.

\section{CONCLUSIONS}

In this work, a conformational and vibrational study of the well-known chemotherapeutic drug cisplatin was undertaken, by quantum mechanical calculations. Different theoretical methodologies-HF, DFT, and MP2 - and all-electron basis sets, to describe the nonmetal atoms were tested. The best combination between the theory level and the AE basis was determined, in order to achieve an accurate representation of this kind of metal complexes, with an optimal compromise between accuracy and computational cost.

The best compromise between accuracy and computational demands was achieved by the DFT mPW1PW approach coupled to the $6-31 \mathrm{G}(d)$ basis set [mPW1-AE1; the $\mathrm{Pt}$ atom being represented by the relativistic effective core potential LANL2DZ (using an intermediate integration grid of 75 radial shells and 302 angular points per shell)].

The result disagrees from the previously reported work of Pavankumar et al., ${ }^{25}$ which refers all three geometries as real minima in the cDDP potential energy surface, independently of the theoretical level considered. In fact, depending on the theoretical approach, a sole minimum (cDDP1) or two different conformers (cDDP1 and cDDP2) are predicted for cisplatin, varying in the relative orientation of the $\mathrm{NH}_{3}$ ligands.

The assignments of the cDDP experimental Raman and IR spectra to be found in the literature are still rather incomplete. ${ }^{25,26,45-47}$ The present theoretical study allows fulfilling this gap, by yielding a complete assignment of the vibrational spectra of this anticancer drug. The best frequency scaling model and scaling factors were determined, keeping in mind that transferability to the study of other $\mathrm{Pt}$ complexes is envisaged. This will hopefully allow us to per- form thorough conformational analysis for these types of systems (at an optimal computational cost), in view of gathering precious knowledge on their molecular properties. This is essential for understanding the mechanisms underlying antineoplastic activity, in order to develop novel metal-based anticancer agents.

\section{ACKNOWLEDGMENTS}

The authors acknowledge financial support from the Portuguese Foundation for Science and Technology-Unidade de Química-Física Molecular, Research Project POCTI/47256/ QUI/2002 (cofinanced by the european community fund FEDER) and Ph.D. fellowship SFRH/BD/17493/2004 (S.M.F.). Laboratório Associado CICECO (University of Aveiro, Portugal) is also acknowledged for free access to the FT-Raman and FTIR spectrometers.

${ }^{1}$ E. Alessio, E. Iengo, S. Zorzet, A. Bergamo, M. Coluccia, A. Boccarelli, and G. Sava, J. Inorg. Biochem. 79, 173 (2000).

${ }^{2}$ E. Alessio, G. Mestroni, A. Bergamo, and G. Sava, in Metal Ions in Biological Systems, Vol. 42: Metal Complexes in Tumor Diagnosis and as Anticancer Agents CRC, New York (2004), Vol. 42, p. 323.

${ }^{3}$ A. Bergamo, S. Zorzet, B. Gava, A. Sorc, E. Alessio, E. Iengo, and G. Sava, Anti-Cancer Drugs 11, 665 (2000).

${ }^{4}$ G. H. Bulluss, K. M. Knott, E. S. F. Ma, S. M. Aris, E. Alvarado, and N. Farrell, Inorg. Chem. 45, 5733 (2006).

${ }^{5}$ S. Choi, C. Filotto, M. Bisanzo, S. Delaney, D. Lagasee, J. L. Whitworth, A. Jusko, C. R. Li, N. A. Wood, J. Willingham, A. Schwenker, and K. Spaulding, Inorg. Chem. 37, 2500 (1998).

${ }^{6}$ O. Delalande, J. Malina, C. Hofr, O. Novakova, G. Natile, J. Kozelka, and V. Brabec, J. Inorg. Biochem. 86, 201 (2001).

${ }^{7}$ B. A. J. Jansen, J. van der Zwan, J. Reedijk, H. den Dulk, and J. Brouwer, Eur. J. Inorg. Chem. 9, 1429 (1999).

${ }^{8}$ L. R. Kelland, S. Y. Sharp, C. F. O'Neill, F. I. Raynaud, P. J. Beale, and I. R. Judson, J. Inorg. Biochem. 77, 111 (1999).

${ }_{9}^{9}$ B. Lippert, Coord. Chem. Rev. 182, 263 (1999).

${ }^{10}$ C. Manzotti, G. Pratesi, E. Menta, R. Di Domenico, E. Cavalletti, H. H. Fiebig, L. R. Kelland, N. Farrell, D. Polizzi, R. Supino, G. Pezzoni, and F. Zunino, Clin. Cancer Res. 6, 2626 (2000).

${ }^{11}$ V. Marini, P. Christofis, O. Novakova, J. Kasparkova, N. Farrell, and V. Brabec, Nucleic Acids Res. 33, 5819 (2005).

${ }^{12}$ M. P. M. Marques, T. Girao, M. C. P. De Lima, A. Gameiro, E. Pereira, and P. Garcia, Biochim. Biophys. Acta 1589, 63 (2002).

${ }^{13}$ G. Mestroni, E. Alessio, A. S. Santi, S. Geremia, A. Bergamo, G. Sava, A. Boccarelli, A. Schettino, and M. Coluccia, Inorg. Chim. Acta 273, 62 (1998).

${ }^{14}$ R. E. Morris, R. E. Aird, P. D. Murdoch, H. M. Chen, J. Cummings, N. D. Hughes, S. Parsons, A. Parkin, G. Boyd, D. I. Jodrell, and P. J. Sadler, 
J. Med. Chem. 44, 3616 (2001).

${ }^{15}$ P. D. Murdoch, N. A. Kratochwil, J. A. Parkinson, M. Patriarca, and P. J. Sadler, Angew. Chem., Int. Ed. 38, 2949 (1999).

${ }^{16}$ C. Navarro-Ranninger, F. Zamora, J. M. Perez, I. Lopezsolera, S. Martinezcarrera, J. R. Masaguer, and C. Alonso, J. Inorg. Biochem. 46, 267 (1992).

${ }^{17}$ G. B. Onoa, V. Moreno, E. Freisinger, and B. Lippert, J. Inorg. Biochem. 89, 237 (2002).

${ }^{18}$ G. Pratesi, P. Perego, D. Polizzi, S. C. Righetti, R. Supino, C. Caserini, C. Manzotti, F. C. Giuliani, G. Pezzoni, S. Tognella, S. Spinelli, N. Farrell, and F. Zunino, Br. J. Cancer 80, 1912 (1999).

${ }^{19}$ A. G. Quiroga, J. M. Perez, C. Alonso, C. Navarro-Ranninger, and N. Farrell, J. Med. Chem. 49, 224 (2006).

${ }^{20}$ T. Servidei, C. Ferlini, A. Riccardi, D. Meco, G. Scambia, G. Segni, C. Manzotti, and R. Riccardi, Eur. J. Cancer 37, 930 (2001).

${ }^{21}$ N. J. Wheate and J. G. Collins, Coord. Chem. Rev. 241, 133 (2003).

${ }^{22}$ M. Schmitt and J. Popp, J. Raman Spectrosc. 37, 20 (2006).

${ }^{23}$ M. M. Nolasco, A. M. Amado, and P. J. A. Ribeiro-Claro, ChemPhysChem 7, 2150 (2006).

${ }^{24}$ D. Michalska and R. Wysokinski, Chem. Phys. Lett. 403, 211 (2005).

${ }^{25}$ P. N. V. Pavankumar, P. Seetharamulu, S. Yao, J. D. Saxe, D. G. Reddy, and F. H. Hausheer, J. Comput. Chem. 20, 365 (1999).

${ }^{26}$ R. Wysokinski and D. Michalska, J. Comput. Chem. 22, 901 (2001).

${ }^{27}$ G. H. W. Milburn and M. R. Truter, J. Chem. Soc. A 1966, 1609.

${ }^{28}$ M. J. Frisch, G. W. Trucks, H. B. Schlegel, G. E. Scuseria, M. A. Robb, J. R. Cheeseman, J. A. Montgomery, Jr., T. Vreven, K. N. Kudin, J. C. Burant, J. M. Millam, S. S. Iyengar, J. Tomasi, V. Barone, B. Mennucci, M. Cossi, G. Scalmani, N. Rega, G. A. Petersson, H. Nakatsuji, M. Hada, M. Ehara, K. Toyota, R. Fukuda, J. Hasegawa, M. Ishida, T. Nakajima, Y Honda, O. Kitao, H. Nakai, M. Klene, X. Li, J. E. Knox, H. P. Hratchian, J. B. Cross, V. Bakken, C. Adamo, J. Jaramillo, R. Gomperts, R. E. Stratmann, O. Yazyev, A. J. Austin, R. Cammi, C. Pomelli, J. W. Ochterski, P. Y. Ayala, K. Morokuma, G. A. Voth, P. Salvador, J. J. Dannenberg, V. G. Zakrzewski, S. Dapprich, A. D. Daniels, M. C. Strain, O. Farkas, D. K. Malick, A. D. Rabuck, K. Raghavachari, J. B. Foresman, J. V. Ortiz, Q. Cui, A. G. Baboul, S. Clifford, J. Cioslowski, B. B. Stefanov, G. Liu,
A. Liashenko, P. Piskorz, I. Komaromi, R. L. Martin, D. J. Fox, T. Keith, M. A. Al-Laham, C. Y. Peng, A. Nanayakkara, M. Challacombe, P. M. W. Gill, B. Johnson, W. Chen, M. W. Wong, C. Gonzalez, and J. A. Pople, Gaussian 03, Revision D.01, Gaussian, Inc., Wallingford, CT, 2004.

${ }^{29}$ P. J. Hay and W. R. Wadt, J. Chem. Phys. 82, 299 (1985).

${ }^{30}$ A. W. Ehlers, M. Bohme, S. Dapprich, A. Gobbi, A. Hollwarth, V. Jonas, K. F. Kohler, R. Stegmann, A. Veldkamp, and G. Frenking, Chem. Phys. Lett. 208, 111 (1993).

${ }^{31}$ H. Chermette, Coord. Chem. Rev. 180, 699 (1998).

${ }^{32}$ A. D. Becke, Phys. Rev. A 38, 3098 (1988).

${ }^{33}$ C. T. Lee, W. T. Yang, and R. G. Parr, Phys. Rev. B 37, 785 (1988).

${ }^{34}$ C. Adamo and V. Barone, J. Chem. Phys. 108, 664 (1998).

${ }^{35}$ J. P. Perdew, K. Burke, and Y. Wang, Phys. Rev. B 54, 16533 (1996).

${ }^{36}$ A. P. Scott and L. Radom, J. Phys. Chem. 100, 16502 (1996).

${ }^{37}$ See EPAPS document No. E-JCPSA6-127-311738. This document can be reached through a direct link in the online article's HTML reference section or via the EPAPS homepage (http://www-aip-org/pubservs/ epaps.html).

${ }^{38}$ L. A. E. Batista de Carvalho, M. P. M. Marques, and J. Tomkinson (unpublished).

${ }^{39}$ E. F. C. Byrd, C. D. Sherrill, and M. Head-Gordon, J. Phys. Chem. A 105, 9736 (2001)

${ }^{40}$ B. Galabov, Y. Yamaguchi, R. B. Remington, and H. F. Schaefer, J. Phys. Chem. A 106, 819 (2002).

${ }^{41}$ M. D. Halls, J. Velkovski, and H. B. Schlegel, Theor. Chem. Acc. 105, 413 (2001).

${ }^{42}$ X. Zhou and R. Liu, Vib. Spectrosc. 12, 65 (1996).

${ }^{43}$ X. Zhou, S. J. Mole, and R. Liu, Vib. Spectrosc. 12, 73 (1996).

${ }^{44}$ X. Zhou, C. J. M. Wheeless, and R. Liu, Vib. Spectrosc. 12, 53 (1996).

${ }^{45}$ H. Baranska, J. Kuduk-Jaworska, and J. Baran, Asian J. Phys. 7, 265 (1998).

${ }^{46}$ J. Hiraishi, I. Nakagawa, and T. Shimanou, Spectrochim. Acta, Part A 24, 819 (1968).

${ }^{47}$ K. Nakamoto, P. J. McCarthy, J. Fujita, R. A. Condrate, and G. T. Behnke, Inorg. Chem. 4, 36 (1965). 